\title{
Consumption of dairy products and the 15-year incidence of age-related macular degeneration
}

\author{
Bamini Gopinath ${ }^{1}$, Victoria M. Flood ${ }^{2}$, Jimmy C. Y. Louie ${ }^{2}$, Jie Jin Wang ${ }^{1,3}$, George Burlutsky ${ }^{1}$, \\ Elena Rochtchina ${ }^{1}$ and Paul Mitchell ${ }^{1 *}$ \\ ${ }^{1}$ Centre for Vision Research, Department of Ophthalmology and Westmead Millennium Institute, University of Sydney, \\ Westmead Hospital, Hawkesbury Road, Westmead, NSW 2145, Australia \\ ${ }^{2}$ Faculty of Health and Behavioural Sciences, University of Wollongong, Sydney, NSW, Australia \\ ${ }^{3}$ Department of Ophthalmology, Centre for Eye Research Australia, University of Melbourne, Melbourne, Australia
}

(Submitted 14 May 2013 - Final revision received 30 September 2013 - Accepted 11 November 2013 - First published online 6 February 2014)

\section{Abstract}

Habitual consumption of dairy products has been shown to play an important role in the prevention of several chronic diseases. We aimed to prospectively assess the relationship between the change in dairy product consumption (both regular fat and low/reduced fat) and the 15-year incidence of age-related macular degeneration (AMD). In the Blue Mountains Eye Study, 2037 participants aged 49 years or above at baseline were re-examined at follow-up in 1997-9, 2002-4 and/or 2007-9. AMD was assessed from retinal photographs. Dietary data were collected using a semi-quantitative FFQ, and servings of dairy product consumption calculated. Over the 15-year follow-up, there were 352, 268 and eighty-four incident cases of any, early and late AMD, respectively. After adjusting for age, sex, current smoking, white cell count and fish consumption, a significant linear trend $(P$ for trend $=0.003)$ was observed with decreasing consumption of total dairy foods and the 15-year incidence of late AMD, comparing the lowest $v$. highest quintile of intake (OR 2.80, $95 \%$ CI 1.21, 3.04). Over the 15 years, decreased consumption of reduced-fat dairy foods was associated with an increased risk of incident late AMD, comparing the lowest to highest quintile of intake (OR $3 \cdot 10,95 \%$ CI $1 \cdot 18,8 \cdot 14, P$ for trend=0.04). Decreasing total dietary Ca intake over the 15 years was also associated with an increased risk of developing incident late AMD (multivariable-adjusted $P$ for trend=0.03). A lower consumption of dairy products (regular and low fat) and Ca was independently associated with a higher risk of developing incident late AMD in the long term. Additional cohort studies are needed to confirm these findings.

\section{Key words: Age-related macular degeneration: Calcium: Dairy products: Blue Mountains Eye Study: Reduced fat foods}

Age-related macular degeneration (AMD) is a progressive, chronic disease of the central retina and is the leading cause of vision loss in older people worldwide ${ }^{(1)}$. Major risk factors include cigarette smoking, nutritional factors, CVD and genetic markers, including genes that regulate complement, lipid, angiogenic and extracellular matrix pathways ${ }^{(2)}$. To date, very few epidemiological data on modifiable risk factors are consistent and well validated, so that the only well-established modifiable risk factor is smoking ${ }^{(2,3)}$. Better understanding of modifiable risk factors will facilitate to identify and change at-risk behaviour, and help in implementing preventive strategies early in the disease process ${ }^{(3)}$.

There is accumulating evidence from published studies demonstrating that regular consumption of dairy foods could counteract the risk of obesity, the metabolic syndrome, type 2 diabetes, hypertension and $\mathrm{CVD}^{(4-7)}$. Given that systemic risk factors such as obesity, cardiovascular risk factors (e.g. hypertension) and CVD have also been linked with $\mathrm{AMD}^{(2)}$, there is a potential for regular consumption of dairy products to modify the risk of AMD in older adults. Moreover, regular consumption of dairy products has been demonstrated to confer protection against inflammation, oxidative stress and endothelial dysfunction ${ }^{(6,8)}$. Given that inflammation and endothelial dysfunction have been implicated in the pathogenesis of $\mathrm{AMD}^{(9-11)}$, these could be potential pathways by which dairy product consumption modifies the risk of developing AMD lesions.

To our best knowledge, there are no epidemiological data on the relationship between habitual consumption of dairy products and the risk of incident AMD. In the present cohort study of

Abbreviations: AMD, age-related macular degeneration; ARMS2, age-related maculopathy susceptibility gene 2; BMES, Blue Mountains Eye Study; CFH, complement factor $\mathrm{H}$.

*Corresponding author: P. Mitchell, fax +6129845 6117, email paul.mitchell@sydney.edu.au 
adults aged 49 years and over, we aimed to answer the following key questions: (1) is regular consumption of dairy products (comprising three primary dairy products consumed in the present study population - milk, cheese and yogurt) and total $\mathrm{Ca}$ intake at baseline prospectively associated with the 15-year incidence of AMD, independent of potential confounders such as smoking, white cell count and fish consumption? and (2) do any associations found differ by the type of dairy foods consumed, i.e. regular/high-fat compared with low/reduced-fat dairy products?

\section{Methods \\ Study population}

The Blue Mountains Eye Study (BMES) is a population-based cohort study of common eye diseases and other health outcomes in a suburban Australian population located west of Sydney. Study methods and procedures have been described elsewhere ${ }^{(12)}$. Participants were non-institutionalised residents aged 49 years or older invited to attend a detailed baseline eye examination after a door-to-door census of the study area. Selection bias at baseline was minimised after multiple callback visits, including door-knocking, telephone reminders and letters at recruitment. Baseline examinations of 3654 residents aged $>49$ years were conducted during 1992-4 (BMES-1, 82.4\% participation rate). Surviving baseline participants were invited to attend examinations after 5 (1997-9, BMES-2), 10 (2002-4, BMES-3) and 15 years (2007-9, BMES-4) at which 2334 ( $75 \cdot 1 \%$ of survivors), 1952 ( $75.6 \%$ of survivors) and 1149 (55.4\% of survivors) participants were re-examined, respectively, with complete data. Participants who did not return to the 5-year visit were also invited to the 10- or 15-year visits. The University of Sydney and the Western Sydney Area Human Ethics Committees approved the study, and written informed consent was obtained from all participants at each examination.

\section{Assessment of age-related macular degeneration}

Incidence of AMD was the main outcome, 5, 10 or 15 years later. We took two $30^{\circ}$ stereoscopic colour retinal photographs of the macula of both eyes, which were graded for the presence of early and late AMD using the Wisconsin AMD Grading System ${ }^{(13,14)}$. Inter-grader and intra-grader reliability showed good agreement in identifying individual lesions ${ }^{(15)}$. The detailed methodology of AMD ascertainment in this population has been reported extensively elsewhere ${ }^{(13,14)}$. Incident early AMD was defined as the absence of late AMD and the presence of either (1) large $(>125 \mu \mathrm{m}$ diameter) indistinct soft or reticular drusen or (2) both large distinct soft drusen and retinal pigmentary abnormalities (hyperpigmentation or hypopigmentation) at BMES-2, -3 or -4 in either eye of persons free of early AMD in both eyes at BMES-1 ${ }^{(14)}$. Similarly, incident late AMD was defined as the appearance of neovascular AMD or geographic atrophy at BMES-2, -3 or -4 in either eye of persons without AMD lesions in both eyes at BMES-1 ${ }^{(14)}$. Incident any AMD was defined as having early or late AMD at BMES-2, -3 or -4 . A retinal specialist (P. M.) adjudicated all uncertain retinal pathology and confirmed all late-AMD cases.

\section{Assessment of dairy product consumption}

Dietary data were collected using a 145-item self-administered FFQ, modified for Australian diet and vernacular from an early Willett $F_{F Q}{ }^{(16)}$ and including reference portion sizes. Participants used a nine-category frequency scale to indicate the usual frequency of consuming individual food items during the past year. The FFQ was validated by comparing nutrients from the FFQ with $3 \times 4 \mathrm{~d}$ weighed food records collected over 1 year $(n$ 79) in order to assess seasonal variation. Most nutrient correlations were between 0.50 and 0.60 (e.g. 0.61 for $\mathrm{Ca}$ ) for energy-adjusted intakes, similar to other validated FFQ studies ${ }^{(17,18)}$. A dietitian coded data from the FFQ into a customised database that incorporated the Australian Tables of Food Composition 1990 (NUTTAB90), and follow-up data used NUTTAB95 $5^{(19,20)}$.

Foods listed in the FFQ were categorised into major food categories and subcategories similar to those used for the 1995 Australian National Nutrition Survey ${ }^{(21)}$. Dairy product subcategorisation includes regular milk, reduced fat/skimmed milk, low-fat cheese, regular cheese, reduced-fat dairy dessert (e.g. low-fat yogurt) and medium-fat dairy dessert (e.g. custard and regular yogurt). For the purpose of the present analysis, total dairy products included all of the aforementioned dairy foods; low/reduced-fat dairy products included 'reduced fat/ skimmed milk', 'reduced-fat dairy dessert' and 'low-fat cheese'; while regular-fat dairy products included 'regular milk', 'regular cheese' and 'medium-fat dairy dessert'. Quintiles of dairy product consumption were based on servings of dairy product consumed per $d$. The serving sizes used were $250 \mathrm{~g}$ for milk, $200 \mathrm{~g}$ for yogurt, $250 \mathrm{ml}$ for custards and $40 \mathrm{~g}$ for cheese ${ }^{(4)}$. Among the participants in the lowest quintile of low-fat dairy product consumption ( $\leq 0.00$ servings $/ d$ ), approximately $33 \%$ consumed $\geq 1.53$ servings/d of regular-fat dairy products (i.e. in the highest quintile of regular-fat dairy food intake). Similarly, among the participants in the lowest quintile of regular-fat dairy product consumption $(\leq 0 \cdot 17$ servings/d), approximately $30 \%$ consumed $\geq 1 \cdot 18$ servings/d of low-fat dairy product (i.e. in the highest quintile of low-fat dairy product consumption). Total $\mathrm{Ca}$ intake was assessed from foods consumed only and did not include $\mathrm{Ca}$ intake from dietary supplements.

\section{Assessment of covariates}

Covariates were selected based on previously published data showing significant associations with AMD incidence: smoking $^{(22)}$, white cell count ${ }^{(23)}$, fish consumption ${ }^{(24)}$ and AMD risk alleles ${ }^{(25,26)}$. Information on all covariates was available at baseline (BMES-1) and then at the three subsequent follow-up examinations (BMES-2, -3 and -4). Smoking status was determined from history as never smoker, past smoker and current smoker (which included those who had ceased smoking within the last 12 months). Fasting blood samples 
were also processed for white cell count. We extracted separate data on the frequency of consuming fish, including salmon, tuna and sardines from the FFQ. Fruit and vegetable consumption was also considered as a potential covariate; however, it was not significant in the multivariable model and thus not included in the final parsimonious model. The complement factor $\mathrm{H}$ (CFH) SNP rs1061170 and the agerelated maculopathy susceptibility gene 2 (ARMS2) SNP $r s 10490924$ were genotyped or imputed using genome-wide scan data.

\section{Statistical analyses}

SAS statistical software version $9 \cdot 2$ (SAS Institute, Inc.) was used for analyses, including $t$ tests, $\chi^{2}$ tests and logistic regression. Associations between dairy product consumption (including regular-fat and low/reduced-fat dairy products) and the risk of incident AMD (study outcome) were examined in discrete linear logistic regression models, adjusted for age, sex, current smoking at baseline, white cell count and fish consumption. Given the overlap in regular-fat and low-fat dairy product consumption, all analyses were adjusted for high-fat dairy food intake when the study factor was low-fat dairy food intake, and if the study factor was high-fat dairy food intake, then low-fat dairy food intake was included in the multivariable model. The correlation coefficient between the change in regular-fat dairy intake and low-fat dairy product intake over 15 years was moderate i.e. -0.34 $(P<0.0001)$; hence, it was appropriate to adjust for both regular-fat and low-fat dairy product consumption in the final multivariable model. Supplementary analyses involved further adjustment for the presence of CFH and ARMS2 SNP, rs1061170 and rs10490924, respectively. We analysed dairy product consumption and dietary $\mathrm{Ca}$ intake as quintiles and used the highest quintile as the reference group ${ }^{(27)}$. We used discrete time logistic regression modelling to investigate the associations between quintiles of total, regular-fat and low/reduced-fat dairy product consumption and the risk of incident AMD, where the change in dairy product intake in the 15-year study period was taken into account. Findings from all analyses are expressed as adjusted odds ratios and $95 \%$ confidence intervals.

\section{Results}

Of the 3654 residents examined at baseline BMES, 2900 had baseline dietary data. Of these, 2037 had incident AMD data at BMES-2, -3 or -4 and were included for incidence analyses. The study characteristics of the participants included are shown in Table 1 . The participants in the highest $v$. lowest quintile of total dairy product intake were more likely to consume all types of dairy foods, $\geq 1$ serve/week of fish, fruits and vegetables, and to also have higher dietary intakes of saturated fat and vitamin $\mathrm{B}_{12}$ (Table 1 ). The participants in the highest $v$. lowest quintile of regular-fat dairy product consumption were more likely to be male and to have higher white cell counts, and were more likely to consume saturated fat, vitamin $B_{12}$, vegetables and regular-fat milk, yogurt, cheese and custard (Table S1, available online). However, they were less likely to consume all types of low-fat dairy foods (i.e. skimmed milk, low-fat milk and yogurt). The participants in the highest $v$. lowest quintile of low-fat dairy product intake were less likely to be male, older, current smokers and consume all regular-fat dairy foods (regular-fat milk and yogurt). However, these participants had a higher consumption of vitamin $\mathrm{B}_{12}$, saturated fat, low-fat milk, yogurt and cheese, and fruits, vegetables and fish, and higher white cell counts (Table S2, available online).

Table 2 presents the results from discrete time logistic regression analyses, i.e. associations between the change in dairy food intake and the 15-year incidence of AMD. After multivariable adjustment, decreasing consumption of total dairy foods over the 15 years was associated with an increased risk of developing incident late AMD ( $P$ for trend=0.003), comparing lowest with highest quintiles of intake (OR 2.80, $95 \%$ CI 1.21, 3.04). Over the 15 years, reduced consumption of reduced/low-fat dairy foods was associated with an increased risk of incident late AMD, comparing participants in the lowest $v$. highest quintile (OR 3.10, 95\% CI 1·18, 8.14, $P$ for trend $=0.04)$. The change in dietary Ca intake was inversely associated with the 15-year incidence of late AMD (Table 3). After multivariable adjustment, decreasing intake of total dietary $\mathrm{Ca}$ over the 15 years was associated with an increased risk of developing incident late AMD ( $P$ for trend $=0 \cdot 03$ ). Supplementary analyses involved further adjustment for the presence of risk alleles (CFH-rs1061170 and ARMS2-rs10490924). Of the 2037 participants with complete dietary data and information on incident AMD, 1733 and 1601 had $C F H$ and ARMS2 genotype data, respectively. Additional adjustment for AMD risk alleles did not appreciably change observed estimates, and significant associations still persisted when comparing participants in the lowest $v$. highest quintile of low-fat, regular-fat and total dairy food intakes (OR 3.31, 95\% CI 1.16, 9.44, $P$ for trend=0.06; OR 3.02, $95 \%$ CI $1 \cdot 26,7 \cdot 27, P$ for trend=0.02; OR $3 \cdot 16,95 \%$ CI $1 \cdot 28$, $7 \cdot 79, P$ for trend $=0 \cdot 01$, respectively).

\section{Discussion}

The present novel epidemiological study shows that decreased consumption of total, reduced-fat and regular-fat dairy products, and dietary Ca over 15 years was associated with a modest increase in the risk of developing incident late AMD, irrespective of potential confounders such as age, smoking, white cell count, fish consumption and AMD risk alleles. Significant associations were not observed between intakes of dairy food and total dietary $\mathrm{Ca}$ and the risk of incident any or early AMD over the 15 years.

Comparisons with the present findings are difficult, given the lack of other epidemiological studies that have assessed this relationship. Previous studies have, however, shown a protective effect of habitual dairy product consumption on various health outcomes including the metabolic syndrome, hypertension and $\mathrm{CVD}^{(4-7)}$. Moreover, there is evidence to show that higher dairy product intake substantially suppresses oxidative stress and inflammation among overweight and 
Table 1. Baseline characteristics of the study participants stratified by quintiles of total dairy product consumption

(Mean values and standard deviations; number of participants and percentages; $n$ 2037)

Total dairy product consumption (serves/d)

\begin{tabular}{|c|c|c|c|c|c|c|c|c|c|c|c|}
\hline \multirow{2}{*}{ Characteristics } & \multicolumn{2}{|c|}{ 1st quintile ( $n$ 407) } & \multicolumn{2}{|c|}{ 2nd quintile ( $n$ 408) } & \multicolumn{2}{|c|}{ 3rd quintile ( $n$ 407) } & \multicolumn{2}{|c|}{ 4th quintile ( $n$ 409) } & \multicolumn{2}{|c|}{ 5th quintile ( $n$ 406) } & \multirow[b]{2}{*}{$P^{*}$} \\
\hline & Mean & SD & Mean & SD & Mean & SD & Mean & SD & Mean & SD & \\
\hline Age (years) & $63 \cdot 6$ & 8.4 & 63.8 & 8.4 & $63 \cdot 8$ & 8.4 & $64 \cdot 0$ & $8 \cdot 1$ & $63 \cdot 8$ & $8 \cdot 3$ & 0.97 \\
\hline Sex, male & & & & & & & & & & & 0.06 \\
\hline$n$ & \multicolumn{2}{|c|}{188} & \multicolumn{2}{|c|}{191} & \multicolumn{2}{|c|}{169} & \multicolumn{2}{|c|}{155} & \multicolumn{2}{|c|}{178} & \\
\hline$\%$ & \multirow{2}{*}{\multicolumn{2}{|c|}{$46 \cdot 2$}} & \multicolumn{2}{|c|}{$46 \cdot 8$} & \multicolumn{2}{|c|}{41.5} & \multicolumn{2}{|c|}{37.9} & \multicolumn{2}{|c|}{43.8} & \\
\hline Current smoking & & & & & & & & & & & 0.34 \\
\hline$n$ & \multirow{2}{*}{\multicolumn{2}{|c|}{$\begin{array}{c}60 \\
15 \cdot 0\end{array}$}} & \multirow{2}{*}{\multicolumn{2}{|c|}{$\begin{array}{c}46 \\
11.7\end{array}$}} & \multirow{2}{*}{\multicolumn{2}{|c|}{$\begin{array}{c}45 \\
11.5\end{array}$}} & \multirow{2}{*}{\multicolumn{2}{|c|}{$\begin{array}{c}42 \\
10.5\end{array}$}} & \multirow{2}{*}{\multicolumn{2}{|c|}{$\begin{array}{c}53 \\
13.2\end{array}$}} & \\
\hline$\%$ & & & & & & & & & & & \\
\hline White cell count $\left(\times 10^{9} / /\right)$ & $6 \cdot 3$ & 1.7 & 6.4 & 1.6 & $6 \cdot 3$ & 1.6 & $6 \cdot 4$ & 1.8 & 6.5 & 1.7 & 0.84 \\
\hline Saturated fat $(\mathrm{g} / \mathrm{d})$ & $22 \cdot 3$ & $10 \cdot 7$ & $26 \cdot 8$ & $11 \cdot 2$ & $28 \cdot 0$ & $10 \cdot 2$ & 31.5 & $12 \cdot 8$ & $40 \cdot 0$ & $15 \cdot 7$ & $<0.0001$ \\
\hline Vitamin $B_{12}(\mu \mathrm{g} / \mathrm{d})$ & 4.2 & $2 \cdot 8$ & $5 \cdot 1$ & 3.5 & $5 \cdot 2$ & $3 \cdot 2$ & $6 \cdot 1$ & 4.9 & 7.5 & $3 \cdot 2$ & $<0.0001$ \\
\hline Reduced-fat dairy foods (servings/d) & 0.09 & 0.15 & 0.41 & 0.43 & 0.59 & 0.52 & 1.02 & 0.85 & 1.65 & 1.48 & $<0.0001$ \\
\hline Skimmed milk (servings/d) & 0.03 & 0.09 & 0.14 & 0.31 & 0.22 & 0.38 & 0.43 & 0.73 & 0.78 & 1.18 & $<0.0001$ \\
\hline Low-fat milk (servings/d) & 0.04 & 0.10 & 0.22 & 0.37 & 0.26 & 0.40 & 0.37 & 0.58 & 0.67 & $1 \cdot 11$ & $<0.0001$ \\
\hline Low-fat yogurt (servings/d) & 0.02 & 0.05 & 0.03 & $0 \cdot 10$ & 0.08 & $0 \cdot 18$ & 0.17 & 0.30 & 0.14 & 0.34 & $<0.0001$ \\
\hline Low-fat cheese (servings/d) & 0.01 & 0.03 & 0.02 & 0.06 & 0.03 & 0.08 & 0.04 & 0.13 & 0.05 & $0 \cdot 13$ & $<0.0001$ \\
\hline Regular-fat dairy foods (servings/d) & 0.30 & 0.22 & 0.62 & 0.42 & 0.84 & 0.51 & $1 \cdot 12$ & 0.76 & 1.90 & 1.37 & $<0.0001$ \\
\hline Regular-fat milk (servings/d) & 0.09 & 0.15 & 0.36 & 0.41 & 0.43 & 0.46 & 0.47 & 0.65 & 1.00 & 1.21 & $<0.0001$ \\
\hline Regular-fat yogurt (servings/d) & 0.01 & 0.05 & 0.02 & 0.08 & 0.04 & $0 \cdot 11$ & 0.09 & 0.21 & 0.08 & 0.27 & $<0.0001$ \\
\hline Regular-fat cheese (servings/d) & 0.18 & 0.18 & 0.22 & 0.24 & 0.33 & 0.20 & 0.52 & 0.48 & 0.76 & 0.75 & $<0.0001$ \\
\hline Regular-fat custard (servings/d) & 0.02 & 0.04 & 0.02 & 0.06 & 0.04 & 0.08 & 0.05 & 0.09 & 0.05 & 0.09 & $<0.0001$ \\
\hline Fruits $(g / d)$ & $313 \cdot 0$ & 300 & $320 \cdot 8$ & 272 & $341 \cdot 7$ & 226 & $391 \cdot 3$ & 270 & 399.4 & 250 & $<0.0001$ \\
\hline Vegetables $(g / d)$ & 394.6 & 183 & $435 \cdot 6$ & 189 & $433 \cdot 1$ & 168 & $483 \cdot 2$ & 220 & $483 \cdot 2$ & 187 & $<0.0001$ \\
\hline Fish consumption ( $\geq 1$ serve/week) & 201 & $49 \cdot 8$ & 225 & $55 \cdot 3$ & 251 & $62 \cdot 0$ & 272 & $66 \cdot 8$ & 251 & 63.4 & $<0.0001$ \\
\hline 15-year incidence of AMD & & & & & & & & & & & \\
\hline Any & & & & & & & & & & & 0.87 \\
\hline$n$ & & & & & & & & & & & \\
\hline$\%$ & & & & & & & & & & & \\
\hline Early & & & & & & & & & & & 0.82 \\
\hline$n$ & & & & & & & & & & & \\
\hline$\%$ & & & & & & & & & & & \\
\hline Late & & & & & & & & & & & 0.33 \\
\hline$n$ & & & & & & & & & & & \\
\hline$\%$ & & & & & & & & & & & \\
\hline Presence of one or two risk alleles & & & & & & & & & & & \\
\hline CFH-rs 1061170 & & & & & & & & & & & 0.60 \\
\hline$n$ & & & & & & & & & & & \\
\hline$\%$ & & & & & & & & & & & \\
\hline ARMS2-rs10490924 & & & & & & & & & & & 0.91 \\
\hline$n$ & & & & & & & & & & & \\
\hline$\%$ & & & & & & & & & & & \\
\hline
\end{tabular}

AMD, age-related macular degeneration; $C F H$, complement factor $\mathrm{H}$; $A R M S 2$, age-related maculopathy susceptibility gene 2.

*Unadjusted $P$ values from test of heterogeneity across quintiles. 
Table 2. Associations between the change in dairy product consumption and the 15-year incidence of age-related macular degeneration (AMD) in the Blue Mountains Eye Study during 1992-4 to 2007-9

(Adjusted odds ratios and $95 \%$ confidence intervals; $n$ 2037)

\begin{tabular}{|c|c|c|c|c|c|c|c|}
\hline \multirow[b]{2}{*}{ Dairy product consumption (servings/d) } & \multirow[b]{2}{*}{$n$} & \multicolumn{2}{|c|}{ Incident any AMD ( $n$ 352) } & \multicolumn{2}{|c|}{ Incident early AMD ( $n$ 268) } & \multicolumn{2}{|c|}{ Incident late AMD ( $n$ 84) } \\
\hline & & Adjusted OR & $95 \% \mathrm{Cl}$ & Adjusted OR & $95 \% \mathrm{Cl}$ & Adjusted OR & $95 \% \mathrm{Cl}$ \\
\hline \multicolumn{8}{|l|}{ Total dairy products* } \\
\hline 1st quintile ( $\leq 0.78)$ & 414 & 0.98 & $0.66,1.45$ & 0.77 & $0.49,1.21$ & $2 \cdot 80$ & $1.21,3.04$ \\
\hline 2nd quintile $(0.78-1.21)$ & 410 & 1.04 & $0.71,1.52$ & 0.92 & $0.61,1.39$ & $2 \cdot 59$ & $1 \cdot 13,5.94$ \\
\hline 3rd quintile $(1.21-1.70)$ & 410 & $1 \cdot 19$ & $0.81,1.74$ & $1 \cdot 11$ & $0.73,1.67$ & 1.78 & $0.72,4.41$ \\
\hline 4th quintile $(1.70-2 \cdot 75)$ & 418 & 1.09 & $0.74,1.59$ & 1.08 & $0.72,1.62$ & 1.26 & $0.49,3.28$ \\
\hline 5th quintile $(\geq 2.75)$ & 385 & \multirow{2}{*}{\multicolumn{2}{|c|}{$\begin{array}{r}1.0 \text { (reterence) } \\
0.83\end{array}$}} & 1.0 (reference) & & 1.0 (reference & \\
\hline$P$ for trend & & & & \multicolumn{2}{|c|}{0.20} & \multicolumn{2}{|c|}{0.003} \\
\hline \multicolumn{8}{|l|}{ Reduced/low-fat dairy products ${ }^{*} \dagger$} \\
\hline 1st quintile $(\leq 0.00)$ & 458 & 1.04 & $0.70,1.55$ & 0.91 & $0.59,1.40$ & $3 \cdot 10$ & $1 \cdot 18,8 \cdot 14$ \\
\hline 2nd quintile $(0.02-0.07)$ & 398 & 0.77 & $0.51,1.15$ & 0.63 & $0.40,0.99$ & $2 \cdot 36$ & $0.87,6.44$ \\
\hline 3rd quintile $(0.07-0.79)$ & 377 & 0.88 & $0.60,1.30$ & 0.71 & $0.46,1.08$ & 3.93 & $1.53,10 \cdot 10$ \\
\hline 4th quintile $(0.81-1.18)$ & 422 & 0.93 & $0.64,1.34$ & 0.87 & $0.59,1.28$ & 2.02 & $0.76,5.39$ \\
\hline 5th quintile $(\geq 1 \cdot 18)$ & 382 & 1.0 (reference) & & 1.0 (reference) & & 1.0 (reference & \\
\hline$P$ for trend & & \multicolumn{2}{|l|}{0.85} & \multicolumn{2}{|c|}{0.29} & \multicolumn{2}{|c|}{0.04} \\
\hline \multicolumn{8}{|l|}{ Regular-fat dairy products $¥ \ddagger$} \\
\hline 1st quintile $(\leq 0 \cdot 17)$ & 415 & $1 \cdot 13$ & $0.77,1.66$ & 1.02 & $0.67,1.55$ & $2 \cdot 60$ & $1 \cdot 12,6 \cdot 03$ \\
\hline 2nd quintile $(0.18-0.46)$ & 389 & 0.93 & $0.62,1.39$ & 0.82 & $0.53,1.27$ & 1.84 & $0.78,4.33$ \\
\hline 3rd quintile $(0.47-0.86)$ & 408 & 0.99 & $0.67,1.44$ & 0.83 & $0.55,1.26$ & $2 \cdot 12$ & $0.94,4.80$ \\
\hline 4th quintile $(0.86-1.53)$ & 412 & 0.95 & $0.84,1.08$ & 0.74 & $0.49,1.14$ & 1.59 & $0.71,3.56$ \\
\hline 5th quintile $(\geq 1.53)$ & 413 & 1.0 (reference) & & 1.0 (reference) & & 1.0 (reference & \\
\hline$P$ for trend & & \multicolumn{2}{|c|}{0.40} & \multicolumn{2}{|c|}{0.76} & \multicolumn{2}{|c|}{0.03} \\
\hline
\end{tabular}

${ }^{*}$ Adjusted for age, sex, current smoking, white cell count and fish consumption.

† Further adjusted for change in regular-fat dairy product consumption over 15 years.

$\ddagger$ Further adjusted for change in low-fat dairy product consumption over 15 years.

obese adults $^{(28)}$. Also, prior clinical trials have shown that an increase in dairy food intake results in the suppression of circulating C-reactive protein during weight loss ${ }^{(29)}$. There is evidence for an inflammatory process in $\operatorname{AMD}^{(9,10)}$; hence the protection conferred by dairy foods against the development of late AMD could be at least partly explained by their anti-inflammatory properties. Despite the plausible explanations for the beneficial influence of dairy product consumption on macula health, we advise caution because of the large number of comparisons made in the present study, and hence cannot rule out the possibility that the observed protective effect is due to chance.

The primary components of most dairy products could underlie the observed beneficial effect on chronic conditions such as $\mathrm{AMD}$, including $\mathrm{Ca}$, vitamin $\mathrm{D}, \mathrm{Mg}$, dairy protein and a broad range of fatty acids ${ }^{(6)}$. In the present study, total $\mathrm{Ca}$ intake over the 15 years was inversely associated with the risk of developing incident late AMD. This is biologically plausible as the published literature shows that high-Ca diets can suppress systemic oxidative and inflammatory stress ${ }^{(30)}$. Alternatively, other components of dairy products could confer protection. Vitamin D is one such nutrient; a recent US twin study has shown that a higher intake of vitamin $\mathrm{D}$ was associated with a reduced prevalence of $\mathrm{AMD}^{(31)}$. This inverse association could be due to the anti-inflammatory and/or anti-angiogenic properties of vitamin $\mathrm{D}^{(32)}$. In the BMES, we did not assess vitamin $\mathrm{D}$ intake and so cannot confirm this hypothesis. Alternatively, in our cohort, dairy products contribute to 34 and $29 \%$ of vitamin $\mathrm{B}_{12}$ intake in women and men, respectively. Vitamin $\mathrm{B}_{12}$ could confer protection against $\mathrm{AMD}^{(33)}$, due to its ability to lower serum homocysteine concentrations ${ }^{(33)}$ and reverse endothelial dysfunction ${ }^{(11)}$, both of which have been implicated in AMD pathogenesis. Indeed, participants in the lowest quintile of total dairy product consumption had the lowest mean dietary intake of vitamin $\mathrm{B}_{12}$ (Table 1), which

Table 3. Associations between the change in total $\mathrm{Ca}$ intake and the 15-year incidence of age-related macular degeneration (AMD) in the Blue Mountains Eye Study during 1992-4 to 2007-9

(Adjusted odds ratios and $95 \%$ confidence intervals; $n$ 2037)

\begin{tabular}{|c|c|c|c|c|c|c|c|}
\hline \multirow[b]{2}{*}{ Total Ca intake $(\mathrm{mg} / \mathrm{d})^{*}$} & \multirow[b]{2}{*}{$n$} & \multicolumn{2}{|c|}{ Incident any AMD ( $n$ 352) } & \multicolumn{2}{|c|}{ Incident early AMD (n 268) } & \multicolumn{2}{|c|}{ Incident late AMD ( $n$ 84) } \\
\hline & & Adjusted OR & $95 \% \mathrm{Cl}$ & Adjusted OR & $95 \% \mathrm{Cl}$ & Adjusted OR & $95 \% \mathrm{Cl}$ \\
\hline 1st quintile $(\leq 565 \cdot 1)$ & 428 & $1 \cdot 08$ & $0.73,1.59$ & 0.97 & $0.63,1.49$ & 2.99 & $1 \cdot 23,7 \cdot 25$ \\
\hline 2nd quintile (565.5-746.9) & 394 & 1.00 & $0.67,1.48$ & 0.87 & $0.56,1.34$ & $1 \cdot 87$ & $0.74,4.72$ \\
\hline 3rd quintile (747.1-948.6) & 401 & $1 \cdot 15$ & $0.78,1.69$ & 0.98 & $0.64,1.50$ & $3 \cdot 24$ & $1 \cdot 34,7 \cdot 85$ \\
\hline 4th quintile (948.7-1245.9) & 422 & 1.04 & $0.70,1.53$ & 1.03 & $0.68,1.54$ & $1 \cdot 73$ & $0.66,4.50$ \\
\hline 5th quintile ( $\geq 1247 \cdot 3)$ & 389 & \multicolumn{2}{|c|}{1.0 (reference) } & \multicolumn{2}{|c|}{1.0 (reference) } & \multicolumn{2}{|l|}{1.0 (reference) } \\
\hline$P$ for trend & & \multicolumn{2}{|c|}{0.80} & \multicolumn{2}{|c|}{0.63} & \multicolumn{2}{|c|}{0.03} \\
\hline
\end{tabular}

*Adjusted for age, sex, current smoking, white cell count and fish consumption. 
could have contributed to their increased risk of developing AMD. Further studies are warranted to establish the underlying causal pathways that mediate the observed associations between habitual consumption of dairy products and the risk of late AMD.

The results from the present study show that intakes of dairy food and dietary Ca do not confer any protection against the development of early AMD. The reason for the inconsistent associations observed between dairy food consumption and the different stages of AMD remains unclear. However, the present findings concur with a recent systematic review and meta-analysis which demonstrated that dietary lutein and zeaxanthin intake is not significantly associated with a reduced risk of early AMD, whereas an increase in the intake of these carotenoids may be protective against late $\mathrm{AMD}^{(34)}$. Also, prior data from the BMES have shown that frequent fish consumption reduced the risk of incident late but not early $\mathrm{AMD}^{(25)}$. It is possible that the mechanisms for the development of early AMD (primary prevention) may not be the same as those involved in the progression from early to late AMD (secondary prevention) ${ }^{(34)}$, which could lead to the differential associations being observed with various dietary parameters, including dairy foods. Further longitudinal studies are needed to assess the underlying mechanisms responsible for the differential associations observed with early $v$. late AMD.

The present finding that habitual consumption of regular-fat dairy products is protective against the risk of late AMD could have potential public health implications. This is because new treatments are restricted to the neovascular form and mainly stabilise vision ${ }^{(2)}$. Therefore, it has become imperative to identify and develop potential protective approaches to AMD. The present data suggest a possible strategy to diminish the health and economic burden of AMD by encouraging simple changes in the nutritional status of people at risk of AMD, specifically, by incorporating and maintaining adequate intakes of dairy foods in their diets ${ }^{(35)}$. Nevertheless, because of the small number of incident late-AMD cases, these data are suitable for hypothesis generation. We caution that any dietary recommendations from these data such as an increase in the consumption of dairy products to prevent AMD require further validation before they are incorporated into public health advice ${ }^{(3)}$. Specifically, randomised clinical trials that determine more accurately the potential value of dairy food intake as a means of reducing the risk of developing AMD could be valuable.

The strengths of the present study include its prospective design, long-term follow-up of a stable population-based sample and robust data on major confounders and dietary parameters. Furthermore, the present study uses high-quality stereoscopic retinal photography with validated grading to assess macular conditions, and a detailed side-by-side comparison of the baseline and follow-up photographs to ensure negligible misclassification of incident $\mathrm{AMD}^{(24,36)}$. The present study was limited by the relatively small number of incident late-AMD cases; hence, we might not have had sufficient statistical power to detect an association between dairy product consumption and late-AMD incidence. Residual confounding by other lifestyle parameters is also plausible but is speculative based on our known knowledge of risk factors for AMD incidence in the BMES.

In summary, lower consumption of dairy products in the long term could contribute to a modest increase in the risk of developing incident late AMD. The BMES is an observational study, and we cannot prove a cause-effect link; however, it does highlight a necessity to maintain adequate consumption of dairy foods with age. Further prospective studies of the relationship between dairy product consumption and the risk of AMD lesions are nevertheless required. Additional research is also required to establish the pathway(s) that underlie the ability of dairy products to protect against AMD in older adults.

\section{Supplementary material}

To view supplementary material for this article, please visit http://dx.doi.org/10.1017/S000711451300408X

\section{Acknowledgements}

The BMES was funded by the Australian National Health and Medical Research Council (grant no. 974159, 991407, 211069 and 262120), and Westmead Millennium Institute. B. G. was supported by a Macular Degeneration Foundation and Blackmores Dr Paul Beaumont Fellowship. P. M., J. C. Y. L., V. M. F. and G. B. previously received funding from Dairy Australia on other projects related to the BMES dataset. None of the funders had any role in the design, analysis or writing of this article.

The authors' responsibilities were as follows: B. G. and P. M. contributed to the study concept and design; P. M. contributed to the acquisition of the data; G. B. and E. R. carried out the analysis of the data; B. G., V. M. F., J. C. Y. L., J. J. W., E. R. and P. M. were involved in the interpretation of the data; B. G. contributed to the drafting of the manuscript; B. G., V. M. F., J. C. Y. L., E. R., J. J. W. and PM contributed to the critical revision of the manuscript. All authors read and approved the final manuscript.

B. G., J. C. Y. L., V. M. F., E. R., J. J. W., G. B. and P. M. have no conflicts of interest.

\section{References}

1. Foran S, Wang JJ \& Mitchell P (2003) Causes of visual impairment in two older population cross-sections: the Blue Mountains Eye Study. Ophthalmic Epidemiol 10, 215-225.

2. Lim LS, Mitchell P, Seddon JM, et al. (2012) Age-related macular degeneration. Lancet 379, 1728-1738.

3. Guymer RH \& Chong EW (2006) Modifiable risk factors for age-related macular degeneration. Med J Aust 184, 455-458.

4. Louie JC, Flood VM, Rangan AM, et al. (2013) Higher regular fat dairy consumption is associated with lower incidence of metabolic syndrome but not type 2 diabetes. Nutr Metab Cardiovasc Dis 23, 816-821.

5. Malik VS, Sun Q, van Dam RM, et al. (2011) Adolescent dairy product consumption and risk of type 2 diabetes in middleaged women. Am J Clin Nutr 94, 854-861. 
6. Lamarche B (2008) Review of the effect of dairy products on non-lipid risk factors for cardiovascular disease. J Am Coll Nutr 27, 741S-746S.

7. van Meijl LE \& Mensink RP (2011) Low-fat dairy consumption reduces systolic blood pressure, but does not improve other metabolic risk parameters in overweight and obese subjects. Nutr Metab Cardiovasc Dis 21, 355-361.

8. Gibson RA, Makrides M, Smithers LG, et al. (2009) The effect of dairy foods on CHD: a systematic review of prospective cohort studies. Br J Nutr 102, 1267-1275.

9. Hollyfield JG, Bonilha VL, Rayborn ME, et al. (2008) Oxidative damage-induced inflammation initiates agerelated macular degeneration. Nat Med 14, 194-198.

10. Klein R, Knudtson MD, Klein BE, et al. (2008) Inflammation, complement factor $\mathrm{H}$, and age-related macular degeneration: the Multi-ethnic Study of Atherosclerosis. Ophthalmology 115, 1742-1749.

11. Verhaar MC, Wever RM, Kastelein JJ, et al. (1998) 5-Methyltetrahydrofolate, the active form of folic acid, restores endothelial function in familial hypercholesterolemia. Circulation 97, 237-241.

12. Attebo K, Mitchell P \& Smith W (1996) Visual acuity and the causes of visual loss in Australia. The Blue Mountains Eye Study. Ophthalmology 103, 357-364.

13. Wang JJ, Rochtchina E, Lee AJ, et al. (2007) Ten-year incidence and progression of age-related maculopathy: the Blue Mountains Eye Study. Ophthalmology 114, 92-98.

14. Klein BE, Moss SE, Magli YL, et al. (1989) Optic disc cupping: prevalence findings from the WESDR. Invest Ophthalmol Vis Sci 30, 304-309.

15. Mitchell P, Smith W, Attebo K, et al. (1995) Prevalence of age-related maculopathy in Australia. The Blue Mountains Eye Study. Ophthalmology 102, 1450-1460.

16. Willett WC, Sampson L, Browne ML, et al. (1988) The use of a self-administered questionnaire to assess diet four years in the past. Am J Epidemiol 127, 188-199.

17. Barclay AW, Flood VM, Brand-Miller JC, et al. (2008) Validity of carbohydrate, glycaemic index and glycaemic load data obtained using a semi-quantitative food-frequency questionnaire. Public Health Nutr 11, 573-580.

18. Smith W, Mitchell P, Reay EM, et al. (1998) Validity and reproducibility of a self-administered food frequency questionnaire in older people. Aust $N Z J$ Public Health 22, 456-463.

19. Department of Community Services and Health (1990) NUTTAB9O Nutrient Data Table for Use in Australia. Canberra: Australian Government Publishing Service.

20. Department of Community Services and Health (1995) NUTTAB95 Nutrient Data Table for Use in Australia. Canberra: Australian Government Publishing Service.

21. McLennan WAS (1995) National Nutrition Survey, Confidentialised Unit Record File [4807.0]. Canberra: Australian Bureau of Statistics.

22. Tan JS, Mitchell P, Kifley A, et al. (2007) Smoking and the long-term incidence of age-related macular degeneration: the Blue Mountains Eye Study. Arch Ophthalmol 125, 1089-1095.

23. Shankar A, Mitchell P, Rochtchina E, et al. (2007) Association between circulating white blood cell count and long-term incidence of age-related macular degeneration: the Blue Mountains Eye Study. Am J Epidemiol 165, 375-382.

24. Tan JS, Wang JJ, Flood V, et al. (2009) Dietary fatty acids and the 10-year incidence of age-related macular degeneration: the Blue Mountains Eye Study. Arch Ophthalmol 127, 656-665.

25. Wang JJ, Rochtchina E, Smith W, et al. (2009) Combined effects of complement factor $\mathrm{H}$ genotypes, fish consumption, and inflammatory markers on long-term risk for age-related macular degeneration in a cohort. Am J Epidemiol 169, 633-641.

26. Smailhodzic D, Klaver CC, Klevering BJ, et al. (2012) Risk alleles in CFH and ARMS2 are independently associated with systemic complement activation in age-related macular degeneration. Ophthalmology 119, 339-346.

27. Ulm K (1991) A statistical method for assessing a threshold in epidemiological studies. Stat Med 10, 341-349.

28. Zemel MB, Sun X, Sobhani T, et al. (2010) Effects of dairy compared with soy on oxidative and inflammatory stress in overweight and obese subjects. Am J Clin Nutr 91, 16-22.

29. Zemel MB (2009) Proposed role of calcium and dairy food components in weight management and metabolic health. Phys Sportsmed 37, 29-39.

30. Zemel MB \& Sun X (2008) Dietary calcium and dairy products modulate oxidative and inflammatory stress in mice and humans. J Nutr 138, 1047-1052.

31. Seddon JM, Reynolds R, Shah HR, et al. (2011) Smoking, dietary betaine, methionine, and vitamin $\mathrm{D}$ in monozygotic twins with discordant macular degeneration: epigenetic implications. Ophthalmology 118, 1386-1394.

32. Schleithoff SS, Zittermann A, Tenderich G, et al. (2006) Vitamin D supplementation improves cytokine profiles in patients with congestive heart failure: a double-blind, randomized, placebo-controlled trial. Am J Clin Nutr 83, 754-759.

33. Rochtchina E, Wang JJ, Flood VM, et al. (2007) Elevated serum homocysteine, low serum vitamin $\mathrm{B}_{12}$, folate, and age-related macular degeneration: the Blue Mountains Eye Study. Am J Ophthalmol 143, 344-346.

34. Ma L, Dou HL, Wu YQ, et al. (2012) Lutein and zeaxanthin intake and the risk of age-related macular degeneration: a systematic review and meta-analysis. Br J Nutr 107, $350-359$

35. Mozaffarian D, Cao H, King IB, et al. (2010) Trans-palmitoleic acid, metabolic risk factors, and new-onset diabetes in U.S. adults: a cohort study. Ann Intern Med 153, 790-799.

36. Kaushik S, Wang JJ, Flood V, et al. (2008) Dietary glycemic index and the risk of age-related macular degeneration. Am J Clin Nutr 88, 1104-1110. 